\title{
ICI Reduction using Quadrature Kalman Filter for OFDM System
}

\author{
Abhijeet Bishnu \\ Department of \\ Elex. and Telecomm. \\ SGSITS, Indore, India
}

\author{
Anjana Jain \\ Department of \\ Elex. and Telecomm. \\ SGSITS, Indore, India
}

\author{
Anurag Shrivastava \\ Department of \\ Elex. and Telecomm. \\ SGSITS, Indore, India
}

\begin{abstract}
Orthogonal frequency division multiplexing (OFDM) is a technology for high data rate broadband wireless communication system. It provides high spectral efficiency. The main limitation which degrades the performance of OFDM is inter-carrier interference (ICI), which is caused by Doppler frequency offset and transceiver local oscillator frequency offset. To mitigate ICI effect, a new method of ICI reduction using quadrature Kalman filter (QKF) is proposed and investigated. In this paper, we compared proposed method with pulse shaping and self-cancellation methods of ICI reduction for different digital modulation scheme. For simulation purpose AWGN channel is considered and results show that the proposed method outperforms the existing ICI reduction methods.
\end{abstract}

\section{Keywords:}

ICI, OFDM, QKF, frequency offset.

\section{INTRODUCTION}

In OFDM, the high data rate channel is divided into low data rate sub-channels. These sub-channels are modulated on different subcarriers (SCs). Due to low data rate, the bandwidth of each subchannels is less than the coherence bandwidth of channel. Hence, each sub-channels experience flat fading and leads to receiver design simple [1]-[2].

In presence of frequency offset between transceiver oscillator or Doppler frequency shift, the orthogonality between SCs is lost and causes ICI. ICI degrades the performance of OFDM, so ICI is the main issue of OFDM. Many methods have been proposed to mitigate the ICI in literature, including self - cancellation [3]-[5], pulse shaping [6]-[7] etc.

In this paper, a new method of ICI reduction using QKF is proposed to mitigate the effect of ICI in OFDM system. This method is based on estimation and correction of frequency offset. QKF [8] linearize the estimate process and measurement function using statistical linear regression (SLR) through a set of Gauss - Hermite quadrature points.

The rest of paper is organised as follows. Section 2 describes the ICI analysis and brief review of pulse shaping and self-cancellation techniques of ICI mitigation . In section 3, proposed method is in- vestigated. Simulation results are discussed in section 4 and finally conclude the paper in section 5 .

\section{ANALYSIS OF ICI IN OFDM}

The OFDM system model and ICI analysis followed the same technique as in [9]. In base-band system of OFDM model, the timedomain transmitted signal is given by:

$$
x(n)=\frac{1}{N} \sum_{k=0}^{N-1} X(k) e^{j 2 \pi k n / N}
$$

where $x(n)$ denotes the $n^{\text {th }}$ sample of transmitted signal, $X(k)$ denotes the modulated symbol in $k^{t h}$ subcarrier and $N$ is total number of subcarriers.

The received signal in time-domain is given by:

$$
y(n)=x(n) e^{j 2 \pi n \epsilon / N}+w(n)
$$

where $\epsilon(\Delta f T)$ is normalized frequency offset, $\Delta f$ is the Doppler frequency shift and $T$ denotes the OFDM symbol period. $w(n)$ is additive white Gaussian noise.

The received signal at the $k^{\text {th }}$ subcarrier is given by:

$$
\begin{gathered}
Y(k)=\sum_{l=0}^{N-1} y(n) e^{-j 2 \pi k n / N} \\
=X(k) S(0)+\sum_{l=0, l \neq k}^{N-1} X(l) S(l-k)+W(k)
\end{gathered}
$$

The desired carrier signal and ICI signal is given by first and second term of (3) respectively, $W(k)$ is the FFT of $w(n)$. The ICI components are the interfering signals other than the $k^{t h}$ subcarrier signal. $S(l-k)$ are complex coefficients for the ICI components in received signal and expressed as:

$$
S(l-k)=\frac{\sin (\pi(l+\epsilon-k))}{N \sin (\pi(l+\epsilon-k) / N)} e^{j \pi(1-1 / N)(l+\epsilon-k)}
$$

The number of subcarriers $N$ affects little to ICI total power.

Many methods have been proposed to mitigate the ICI effect like pulse shaping, self-cancellation etc. In OFDM spectrum the subcarriers consist of a main lobe followed by reducing amplitude side lobes. When orthogonality is loss between sub-carriers 
due to frequency offset, these side lobes interfere the main lobe and causes ICI [10]. So the main purpose of pulse shaping is to reduce the amplitude of side lobes and hence reduce the ICI power. Improved sinc power pulse (ISP) [7] is proposed, in which $\exp \left(-a(f T)^{2}\right) \operatorname{sinc}^{n}(f T)$ is used to reduce the amplitude of side lobes. Here $a$ is a design parameter to adjust the amplitude and $n$ is the degree of the sinc function.

In self-cancellation, one data symbol is modulated onto next adjacent subcarrier with predefined weighting coefficients. A new scheme of ICI self-cancellation is proposed [5], in which the symbol allocation is given as $X^{\prime}(k)=X(k), X^{\prime}(k+1)=$ $e^{-j \pi / 2} X^{*}(k)$ (where $X^{*}(k)$ is the conjugate of $X(k)$ ). It means the data symbol modulated at $(k+1)^{t h}$ subcarrier is phase rotated by $-\pi / 2$ of the conjugate of the data symbol modulated at $k^{t h}$ subcarrier.

\section{PROPOSED METHOD OF ICI MITIGATION}

The proposed method of ICI mitigation is based on estimation and correction of frequency offset. In this paper, a new QKF algorithm presented in [8] is used for estimation and correction purpose. This new QKF is simplified version of QKF of Ito and Xiong [11]. Ito and Xiong QKF views filtering problem from numerical integration perspective where as New QKF uses statistical linear regression (SLR) to linearise a non-linear function through a set of Gauss Hermite quadrature points. Calculation of $m$ quadrature points are given in [8] and expressed as: Suppose $J$ is a symmetric tridiagonal matrix with zero diagonal elements $\mathrm{J}_{i, i+1}=\sqrt{i / 2}, \quad 1 \leq$ $i \leq(m-1)$ Then calculate the quadrature points $x_{i}$, where $x_{i}$ is the $i$-th eigenvalue of $J$; and the corresponding weight $\omega_{i}=\left(\nu_{i}\right)_{1}^{2}$, where $\left(\nu_{i}\right)_{1}$ is the first element of the $i$-th normalized eigenvector of $J$.

In proposed method, one preamble is sent before transmitting the data information and it is known to both transmitter and receiver. The non-linear function $f(n)$ can be expressed as: $f(n)=\frac{y(n)}{x(n)}$ It can also be expressed as :

$$
f(n)=e^{j 2 \pi n \epsilon / N}+w(n) / x(n)
$$

The QKF algorithm computes both time update and measurement update steps at each time. The steps of time update and measurement update is given in [8] as:

\subsection{Time Update Steps}

(1) Assume at time $n$, the posterior density function $N\left(\hat{\epsilon}_{n-1 \mid n-1}, P_{n-1 \mid n-1}\right)$ is known. Factorize $\mathrm{P}_{n-1 \mid n-1}=$ $\sqrt{P_{n-1 \mid n-1}}\left(\sqrt{P_{n-1 \mid n-1}}\right)^{*}$ where $\hat{\epsilon}_{n-1 \mid n-1}$ and $P_{n-1 \mid n-1}$ is the mean and covariance of posterior density function.

(2) Evaluate the quadrature points as: $\quad \varepsilon_{l, n-1 \mid n-1}=$ $\sqrt{P_{n-1 \mid n-1}} \xi_{l}+\hat{\epsilon}_{n-1 \mid n-1}$ where $\xi_{l}=\sqrt{2} x_{l}$ with weights $\omega_{l}$.

(3) Evaluate the propagated quadrature points as: $\varepsilon_{l, n \mid n-1}^{\prime}=$ $f\left(\varepsilon_{l, n-1 \mid n-1}, n-1\right)$ where $f($.$) is the process function.$

(4) Estimate the predicted state: $\hat{\epsilon}_{n \mid n-1}=\sum_{l=1}^{m} \omega_{l} \varepsilon_{l, n \mid n-1}^{\prime}$

(5) Estimate the predicted error covariance: $\mathrm{P}_{n \mid n-1}=$ $\sum_{l=1}^{m} \omega_{l} \varepsilon_{l, n \mid n-1}^{\prime} \varepsilon_{l, n \mid n-1}^{\prime *}-\hat{\epsilon}_{n \mid n-1} \hat{\epsilon}_{n \mid n-1}^{*}+Q_{n}$ where $Q_{n}$ is the covariance of process noise.

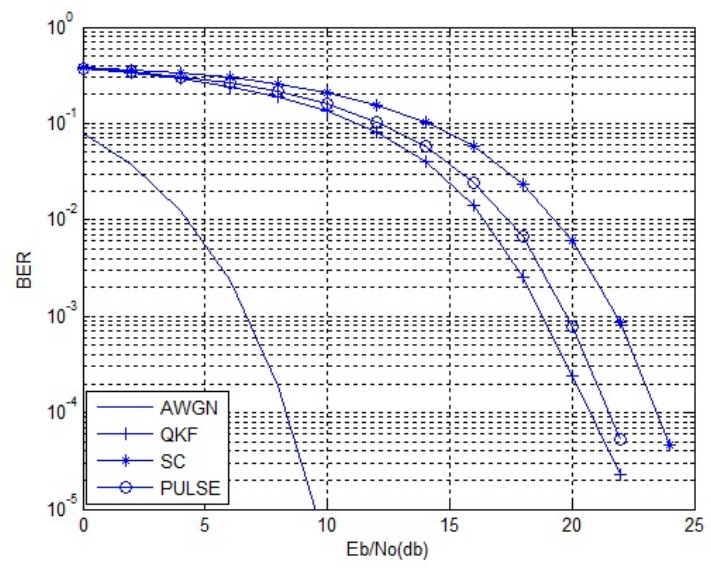

Fig. 1. BER vs $\mathrm{Eb} / \mathrm{No}$ for $\epsilon=0.15$ under BPSK mapping

\subsection{Measurement Update Steps}

(1) Factorize $\mathrm{P}_{n \mid n-1}=\sqrt{P_{n \mid n-1}}\left(\sqrt{P_{n \mid n-1}}\right)^{*}$

(2) Evaluate the quadrature points as: $\varepsilon_{l, n \mid n-1}=\sqrt{P_{n \mid n-1}} \xi_{l}+$ $\hat{\epsilon}_{n \mid n-1}$

(3) Evaluate the propagated quadrature points as: $Z_{l, n \mid n-1}=$ $h\left(\varepsilon_{l, n \mid n-1}, n\right)$ where $h($.$) is the measurement function.$

(4) Estimate the predicted measurement: $\hat{z}_{n \mid n-1}=$ $\sum_{l=1}^{m} \omega_{l} Z_{l, n \mid n-1}$

(5) Estimate the innovation covariance: $\mathrm{P}_{z z, n \mid n-1}=R_{n}+$ $\sum_{l=1}^{m} \omega_{l} Z_{l, n \mid n-1} Z_{l, n \mid n-1}^{*}-\hat{z}_{n \mid n-1} \hat{z}_{n \mid n-1}^{*}$ where $R_{n}$ is the covariance of measurement noise.

(6) Estimate the cross covariance: $\mathrm{P}_{x z, n \mid n-1}=$ $\sum_{l=1}^{m} \omega_{l} \varepsilon_{l, n \mid n-1} Z_{l, n \mid n-1}^{*}-\hat{\epsilon}_{n \mid n-1} \hat{z}_{n \mid n-1}^{*}$

(7) Estimate the Kalman gain: $\mathrm{W}_{n}=P_{x z, n \mid n-1} P_{z z, n \mid n-1}^{*}$

(8) Estimate the updated state: $\hat{\epsilon}_{n \mid n}=\hat{\epsilon}_{n \mid n-1}+W_{n}\left(z_{n}-\hat{z}_{n \mid n-1}\right)$

(9) Estimate the corresponding error covariance: $\mathrm{P}_{n \mid n}=P_{n \mid n-1}-$ $W_{n} P_{z z, n \mid n-1} W_{n}^{*}$

where $(.)^{*}$ is the conjugate of (.). Using the above steps of time update and measurement update, $\epsilon$ is estimated and the effect of ICI in OFDM is effectively eliminated by the compensation of $\hat{\epsilon}$ at the receiver.

\section{RESULTS AND DISCUSSION}

In this section, the performance of proposed method with conventional ICI mitigation method like pulse shaping and selfcancellation in AWGN channel for BPSK and QPSK modulation are compared. We have taken $N=64, N_{p}$ (preamble) $=128$ for simulation and assumed that throughout the transmitting of one block of data $\epsilon$ is constant. For each block of data, time update steps execute one time and measurement update steps execute $N_{p}$ times. Three quadrature points via $\xi_{1}=-\sqrt{3}, \xi_{2}=0$ and $\xi_{3}=\sqrt{3}$, and their corresponding weights, $\omega_{1}=1 / 6, \omega_{2}=2 / 3$ and $\omega_{3}=1 / 6$ respectively (e.g.,see [12] ) are taken.

Figs.1-4 show that as normalised frequency offset increases, the performance of conventional methods of ICI mitigation degrades, it means these methods required large $\mathrm{Eb} / \mathrm{No}$ for same BER as $\epsilon$ increases, so conventional methods are highly sensitive to small 


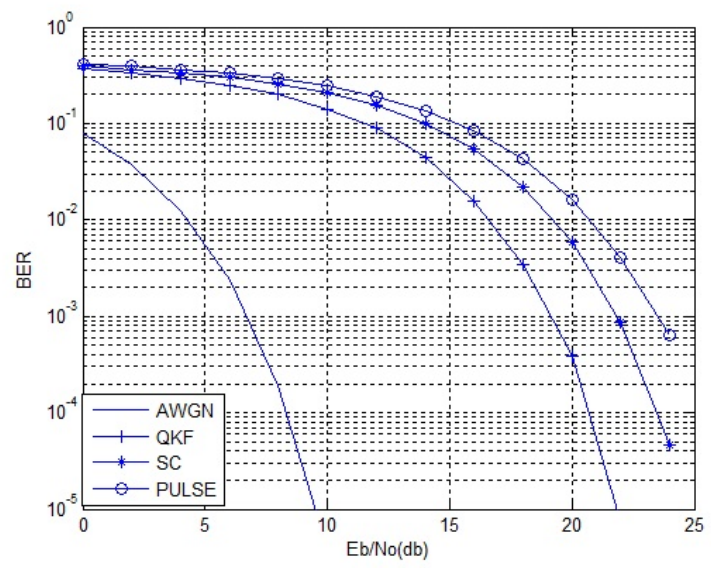

Fig. 2. BER vs Eb/No for $\epsilon=0.3$ under BPSK mapping

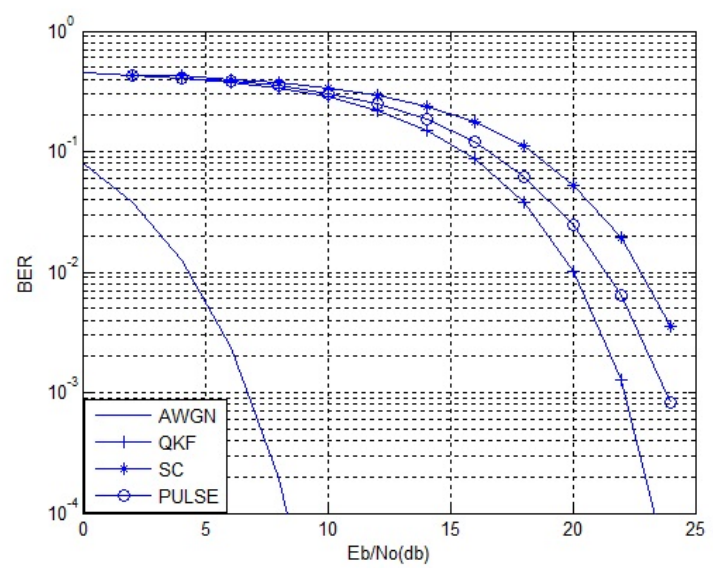

Fig. 3. BER vs Eb/No for $\epsilon=0.15$ under QPSK mapping

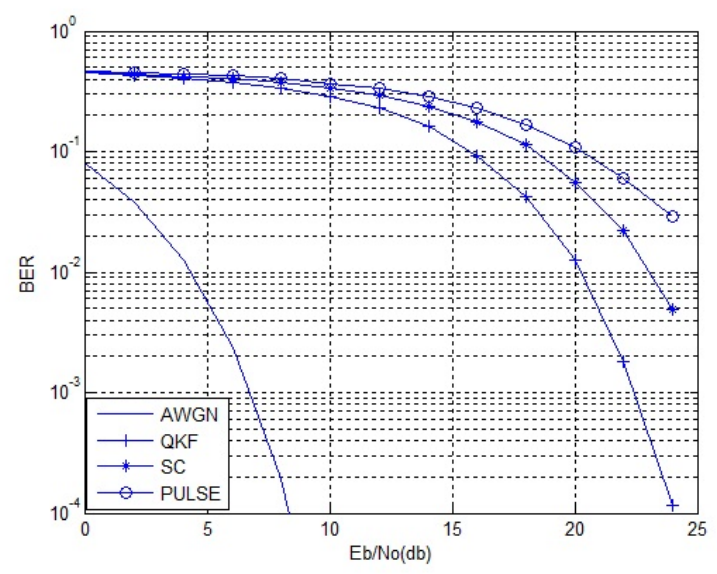

Fig. 4. BER vs $\mathrm{Eb} / \mathrm{No}$ for $\epsilon=0.3$ under QPSK mapping change in $\epsilon$. But the proposed method is less sensetive or insensitive to change in $\epsilon$.

\section{CONCLUSION}

In this paper, a new method of ICI cancellation using QKF has proposed. This method is based on estimation of $\epsilon$ and cancel the effect of ICI by compensation of $\hat{\epsilon}$ at the receiver. It is also less sensitive to change in frequency offset and it outperforms the other ICI cancellation methods as results show. It achieves better BER performance irrespective of $\epsilon$.

\section{REFERENCES}

[1] V. N. Richard and R. Prasad, "OFDM for wireless multimedia communication," Artech House Publisher, London, 2000.

[2] A. Goldsmith, "Wireless communications," Cambridge University Press, New York, 2005.

[3] Y. Zhao and S.G. haggman, Intercarrier interference selfcancellation scheme for OFDM mobile communication systems, IEEE Transaction on Communication, vol. 49, pp. 1185-1191, July 2001

[4] Q. Shi, Y. Fang, M. Wang, A novel ICI self-cancellation scheme for OFDM systems, IEEE WiCom, pp. 1-4, 2009.

[5] A. Bishnu, A. Jain, A. Shrivastava, "A new scheme of ICI self-cancellation in OFDM system", IEEE CSNT 2013, pp. 120-123, April. 2013.

[6] P. Tan, "Reduced ICI in OFDM systems using the Better Than Raised-Cosine pulses", IEEE Communication Letter, vol. 9, no. 5, pp. 135-137, Mar. 2004.

[7] V. Kumbasar and O. Kucur, "ICI reduction in OFDM systems by using improved sinc power pulse", Digital Signal Processing 17, pp. 997-1006, 2007.

[8] I. Arasaratnam, S. Haykin, R. J. Elliott, "Discrete-Time nonlinear filtering algorithms using GaussHermite quadrature", Proceedings of IEEE, vol. 95, no. 5, pp. 953-977, May 2007.

[9] Q. Shi, "ICI Mitigation for OFDM Using PEKF", IEEE Signal Processing Letters, vol. 17, no. 12, pp. 981-984, Dec. 2010.

[10] N. M. Moghaddam and M. Mohebbi, "ICI Reduction Methods in OFDM Systems", Recent Advance in wireless Communication and Networks, ISBN: 978-953-307-274-6, In Tech.

[11] K. Ito and K. Xiong, "Gaussian filters for nonlinear filtering problems", IEEE Transaction on Automation Control, vol. 45, no. 5, pp. 910-927, May 2000.

[12] G. H. Gloub, "Some modified matrix eigenvalue problem ", SIAM Review, vol. 15, pp. 318-334, 1973. 